\title{
Economic Impact of the Application of a Precision Medicine Model (5SPM) on Psychotic Patients
}

\author{
Lorena Carrascal-Laso' \\ Manuel Ángel \\ Franco-Martín' \\ Elena Marcos-Vadillo ${ }^{2}$ \\ Ignacio Ramos-Gallego ${ }^{3}$ \\ Belén García-Berrocal ${ }^{2}$ \\ Eduardo Mayor-Toranzo' \\ Santiago Sánchez-Iglesias ${ }^{4}$ \\ Carolina Lorenzo 4 \\ Alfonso Sevillano-Jiménez' \\ Almudena Sánchez-Martín ${ }^{5}$ \\ María Jesús García-Salgado ${ }^{2}$ \\ María Isidoro-García 2,6 \\ 'Servicio de Psiquiatría, Hospital \\ Provincial de Zamora, IBSAL, Zamora, \\ 4907I, Spain; ${ }^{2}$ Farmacogenética \\ y Medicina de Precisión, Servicio de \\ Bioquímica, Hospital Universitario de \\ Salamanca, IBSAL, Salamanca, 37007, \\ Spain; ${ }^{3}$ Departamento de Fisiología \\ y Farmacología, Universidad de \\ Salamanca, Salamanca, 37007, Spain; \\ ${ }^{4}$ Servicio de Psiquiatría, Hospital \\ Universitario de Salamanca, IBSAL, \\ Salamanca, 37007, Spain; \\ ${ }^{5}$ Pharmacogenetics Unit, Pharmacy \\ Department, University Hospital Virgen \\ de las Nieves, UGC Provincial de \\ Farmacia de Granada, Granada, I80I4, \\ Spain; ${ }^{6}$ Departamento de Medicina, \\ Universidad de Salamanca, Salamanca, \\ 37007, Spain
}

Correspondence: Manuel Ángel FrancoMartín

Email mfrancom@saludcastillayleon.es
Background: Schizophrenia is a severe mental disorder that often manifests within the first three decades of life. Its prognosis is uncertain and may result in a prolonged treatment that could extend throughout the entire lifespan of the patient. Antipsychotic drugs are characterized by a high interindividual variability when considering therapeutic effect and emergence of adverse effects. Such interindividual variability is thought to be associated primarily with pharmacokinetic matters.

Objective: The objective of this study was to evaluate the economic impact of the application of the 5-Step Precision Medicine model (5SPM), an approach based on the pharmacogenetic analysis of the primary genes involved in the metabolism of the therapy for each patient, restructuring treatment as necessary.

Patients and Methods: One hundred eighty-eight psychiatry patients were analysed for single nucleotide polymorphisms on genes CYP1A2, CYP2B6, CYP2C9, CYP2C19, CYP2D6, CYP3A5 and ABCB1. Information on patients' diagnosis, pharmacotherapy, and hospitalizations was collected.

Results: We achieved a cost-benefit ratio of 3.31-3.59 with a reduction of direct cost (hospitalizations plus pharmacotherapy) with a reduction of total cost in $67 \%$ of the patients who underwent the clinical intervention.

Conclusion: A rational Precision Medicine-based approach to psychiatric patients could result in a reduction on number of drugs required to control exacerbations, and the underlying pathologies, reducing the risk of adverse effects and improving adherence to treatment, leading to a potential decrease in direct costs. This methodology has been shown to be costdominant and, being based on a pharmacogenetic analysis, it has a lifelong nature, as the data obtained can be applied to other medical disciplines.

Keywords: pharmacogenetics, pharmacoeconomics, psychotic disorders, cytochrome P-450, antipsychotics

\section{Introduction}

Psychotic disorders encompass a number of serious illnesses requiring complex interdisciplinary treatment grounded in antipsychotic medication. According to the World Health Organization (WHO), schizophrenia affects 20 million people throughout the world. In 2017, the prevalence and incidence of schizophrenia reached 19.78 million and 1.14 million, respectively. ${ }^{1}$ One of the biggest problems that emerges when facing this type of diagnosis, known to be a long-term, serious mental illness, is the fact that the condition often manifests during the first years of the patient's working life. This may significantly impact that person's productivity, even making it impossible to develop a career during what is effectively the most 
productive years of life, further aggravating both the economic burden placed on family members (the impact on their professional life, the need to provide dedicated care and attention to the patient) as well as the emotional burden (involving more social and economic expense driven by psychological deterioration and leading even to abandonment of the patient by the family members). ${ }^{2,3}$

Antipsychotic treatment is necessary for this type of diagnosis, even though not all patients receive the same therapeutic benefits. Moreover, these substances are linked to a high number of adverse effects, which could lead to greater costs as it would become necessary to treat the adverse effects and manage cases that resist treatment. ${ }^{4-6}$ While attempting to solve the more complex cases, it is common for prescribers to opt for polytherapy, which is associated with a higher risk of adverse effects and subsequent hospital stays. It has been suggested that the schizophrenic patient will generate a high economic cost, mainly driven by indirect costs. Given that the criteria with which annual costs are calculated are not bound by a specific standard, in some cases resulting in the overestimation of direct costs and in a disparity of the outcome obtained from different studies of the same population, the total costs associated with schizophrenia vary between 94M (Puerto Rico, 1994, 0.09\% of the Gross Domestic Product (GDP)) and 102,396M (US, 1991, 0.5\% of the GDP) in 2013-USD. Estimates of direct and indirect costs during the interval vary between 29M (Sweden, 2013, $0.02 \%$ of the GDP) and $29,279 \mathrm{M}$ (US, 2002, $0.50 \%$ of the GDP), and between 63M (Puerto Rico, 1994, 0.09\% of the GDP) and 70,597M (US, 1991, 0.5\% of the Gross Domestic Product), respectively. When analyzing these figures, it is also important to take into account the differences among the healthcare systems of different countries. ${ }^{6}$

The last decade has seen a rise in the study of pharmacogenetics, a discipline based on the analysis of the relationship between certain genes and various drugs. Pharmacogenetic analysis as a strategic tool focused on Precision Medicine is a practice that has expanded into specialized fields such as Oncology. ${ }^{7}$ Knowledge of the patient's pharmacogenotype has proved useful when considering certain treatments based on the genetic nature of certain pathologies and has in some cases been able to reduce the costs associated with hospitalizations, adverse effects and pharmacotherapies not adjusted to the pharmacokinetic and/or pharmacodynamic of the patient. ${ }^{8-18}$ There is some evidence to suggest that controlling the dose of various drugs guided by the patient's genotype could reduce the costs of morbidity and mortality associated with drugs that have, in the United States alone, exceeded \$177B. ${ }^{19}$

There have not been many studies to date in the field of Psychiatry that analyze the cost-effectiveness of the application of a pharmacogenetic analysis. ${ }^{20-27}$ It should be noted that, until 2014 , only $27 \%$ of pharmacogenetic studies included an economic analysis of medical intervention. ${ }^{28}$ Despite knowledge of the possible alteration of the plasma levels for the drugs used in psychiatric practice (anti depressives, antipsychotics, mood stabilizers, etc.), associated with genetic variance among genes forming part of the CYP450 superfamily, or the genes related to their transport and distribution (ABCB1), as well as variance in their effectiveness regarding therapeutic targets (5HTR, DDR, LAT, etc.), a cost-benefit analysis applied to this type of strategy remains debatable and is not yet established..$^{20-23,29,30}$ This approach is thought to require a detailed and careful study of the specific circumstances of the patient, as well as the cost of the strategies employed. However, among those pharmacogenetic studies accompanying an economic study, more than $50 \%$ were cost-effective or cost-dominant. Those that were not could qualify by merely applying a small reduction in the technical costs employed. ${ }^{28}$

The primary aim of this study is to evaluate the economic impact that could occur, whether a reduction in pharmaceutical costs or in hospital stays, by implementing a 5SPM precision medicine model based on the pharmacogenetic analysis of patients diagnosed with a severe and long-term mental illness treated with antipsychotics in polytherapy.

\section{Patients and Methods}

For the purposes of the present study, we used a cohort of 188 patients from the psychiatric ward of three public hospitals belonging to the public healthcare service of the Community of Castile and Leon (Castile and Leon Health Services, SACYL), the Health Centre Complex of Zamora (Zamora, Spain), the University Hospital of Salamanca (Salamanca, Spain) and the Rio Hortega University Hospital (Valladolid, Spain). The study was conducted in accordance with the Declaration of Helsinki and was approved by the Ethics Committee of the University Hospital of Salamanca (CEIC ref.: 107/ 12/2016). The patients included in the study provided written informed consent to perform a pharmacogenetic analysis in compliance with the Ethics Committee of the 
University Hospital of Salamanca, where the analyses were carried out. The inclusion criteria were as follows: (1) have a serious, long-term mental illness (2) with poor clinical response to the previously applied treatment that (3) eventually resulted in polypharmacy. Patients who had not received antipsychotic therapy or who were younger than 16 years of age were excluded from the study. Mental illness is considered to be serious and prolonged when its diagnosis involves non-organic psychoses, with a length period and duration of treatment greater than two years, and a moderate to serious impact on work, social and family obligations.

Information was collected on the patients' diagnosis, current psychopharmacotherapy, hospitalizations and total days hospitalized, and the dose/day used, in addition to their age, gender, and pharmacological history. Pharmacological Costs were calculated by using the SRP obtained from the AEMPS/Vademecum Spanish databases, the dose pricing (IM presentations), or by inferring the $€ / \mathrm{mg}$ ratio (oral presentations) from the standard presentation. Hospitalization costs were provided by the Regional Health Administration (SACYL).

The method used in the study was the 5-Step Precision Medicine (5SPM) method as described in Carrascal-Laso et al. ${ }^{31}$ PGx testing was performed using the AmpliChip CYP450 Test (Roche Molecular Diagnostics, Pleasanton, CA, USA), ${ }^{32}$ the Autogenomics platform, MassARRAY 4.2 (Agena) and probe-based assays using the LightCycler platform (Roche Diagnostics). PGx testing was performed following the directives of the European Molecular Genetics Management Network for DNA handling, with the requisite controls. The application of quality norms followed the UNE-EN-ISO 15189:2007 Normative in the Accredited Section of Molecular Genetics and Pharmacogenetics of the Clinical Biochemistry Service of the University Hospital in Salamanca. The genes studied were those encoding enzymes 1A2, 2B6, 2C9, 2C19, 2D6, 3A4, 3A5 of the CYP450 cytochrome family, and ABCB1, which encodes an ATP Binding Cassette transporter.

In order to compare direct economic costs associated before and after the application of the 5SPM model, hospitalizations and pharmacological information were recorded between January 1, 2013 and December 31, 2019, with June 30, 2016 designated as the cut-off point (when the majority of patients were PGx-tested).

\section{Results}

\section{Demographic Data}

The present study concerns a descriptive retrospective study that reports the results for a total of 188 patients from whom clinical data were obtained between 2013 and 2019. The median age of the participants in the study is 47.21 ( \pm 12.93$)$ years of age, with $59.58 \%$ of the participants being women $(n=112)$. The distribution according to diagnosis is presented in Table 1; the most common diagnosis is schizophrenia (DSM-V, F20.?), which was present in $67.02 \%$ of the cases $(n=126)$.

\section{Genotype}

Pharmacogenetic analysis was performed on genes CYP1A2 (allele *1F), CYP2B6 (allele *6), CYP2C9 (alleles *2, *3), CYP2C19 (*2, *4, *17), CYP2D6 (alleles $* 2, * 3, * 4, * 5, * 6, * 7, * 8, * 9, * 10, * 12, * 14, * 17, * 29$, $* 41, * 46)$, CYP3A4 (allele *1B), CYP3A5 (allele *3C) ${ }^{33}$ and ABCB1 (rs10456423435 C>T). The analysis was performed in the Pharmacogenetic and Precision Medicine Unit of the University Hospital of Salamanca. Table 2 indicates the frequency of the appearance of the estimated phenotypes based on the detection of the different alleles present in the sample. Notable in terms of the primary metabolic pathways of the antipsychotics used in the

Table I Demographic Data

\begin{tabular}{|c|c|}
\hline Variable & Value \\
\hline \multicolumn{2}{|l|}{ PATIENTS } \\
\hline $\begin{array}{l}\text { Total number of patients included: } \\
\text { - Average age (range; years) } \\
\text { - Male: Female }(\%)\end{array}$ & $\begin{array}{l}188 \\
47(24-84) \\
59.58: 37.77\end{array}$ \\
\hline \multicolumn{2}{|l|}{ DIAGNOSTIC } \\
\hline DSM-V & n (\%) \\
\hline F03 - Dementia & I $(0.53)$ \\
\hline FI9 - Substance-Related Disorder & $12(6.38)$ \\
\hline F20 - Schizophrenia & $126(67.02)$ \\
\hline F22 - Persistent Delusional Disorder & $2(1.06)$ \\
\hline F23 - Brief and Acute Psychotic Disorder & I $(0.53)$ \\
\hline F25 - Schizoaffective Disorder & $13(6.92)$ \\
\hline F3I - Bipolar Disorder & $25(13.30)$ \\
\hline F33 - Major Depressive Disorder & I $(0.53)$ \\
\hline F60 - Specific Personality Disorders & $2(1.06)$ \\
\hline F6I - Mixed Personality Disorder & I (0.53) \\
\hline F79 - Intellectual Disability & $2(1.06)$ \\
\hline
\end{tabular}

Note: All the pathologies are referred to the official standard nomenclature. Abbreviation: DSM-V, Diagnostic and Statistical Manual of Mental Disorders 5thedition. 
Table 2 Phenotype Relative Abundance

\begin{tabular}{|l|c|c|c|c|c|}
\hline & $\mathbf{n}$ & PM & IM & EM & UM \\
\hline CYPIA2 & 179 & - & - & $13.97 \%(n=25)$ & $86.03 \%(n=154)$ \\
CYP2B6 & 166 & $9.04 \%(n=15)$ & $40.96 \%(n=68)$ & $50 \%(n=83)$ & - \\
CYP2C9 & 183 & $7.10 \%(n=13)$ & $35.52 \%(n=65)$ & $57.38 \%(n=105)$ & - \\
CYP2C19 & 186 & $3.23 \%(n=6)$ & $20.43 \%(n=38)$ & $47.85 \%(n=89)$ & $27.96 \%(n=52)$ \\
CYP2D6 & 183 & $3.78 \%(n=7)$ & $5.95 \%(n=11)$ & $85.95 \%(n=159)$ & $3.24 \%(n=6)$ \\
CYP3A4 & 188 & $1.06 \%(n=2)$ & $7.45 \%(n=14)$ & $91.49 \%(n=172)$ & - \\
CYP3A5 & 187 & $87.85 \%(n=159)$ & $13.81 \%(n=25)$ & $1.66 \%(n=3)$ & - \\
\hline
\end{tabular}

Note: Allele Frequencies of the sample were similar to the NCBI dbSNP ALFA Project Frequencies.

Abbreviations: PM, poor metabolizer; IM, intermediate metabolizer; EM, extensive metabolizer; UM, ultrarapid metabolizer.

sample is that the most prevalent genotype associated with CYP1A2, which was studied in 179 patients, is HI $(86.03 \%, \mathrm{n}=154)$; while the most prevalent genotype associated with CYP2D6 $(\mathrm{n}=183)$ and CYP3A4 $(\mathrm{n}=$ 188) was EM $(85.96, \mathrm{n}=159 ; 91.49 \%, \mathrm{n}=172)$.

\section{Pharmacological Costs}

Although the decision to perform a pharmacogenetic analysis was presented to each patient during their psychiatric consultation, the cut-off date used to establish comparisons with regard to the hospital stays required was 30 June 2016. The period of time studied was 3 years prior and subsequent to that date, since at that moment more than $95 \%$ of the sample had been pharmacogenotyped. Of the 188 included in the study, financial information was obtained for the pharmacotherapy and hospitalizations of 178. Prior to the application of the 5SPM model, the average annual pharmaceutical cost associated with each patient was $2887 €$ per year $(513,805.10 €$ per year in total). With the application of the 5SPM model, a reduction of $9.9 \%$ (287€ per patient per year, 51,187.02€ per year in total) was achieved, resulting in an average of $2598 €$ per patient per year $(462,618.92 €$ per year). Scaled over the three and a half years prior and subsequent to the pharmacogenotype (PGx), the antipsychotic treatment of the patients in the study between 2013 and 2016 involved a cost of $1,798,320.80 €$, with an average cost per patient of $10,102.93 €$, which was reduced to $1,619,166.20 €$, or $9096.44 €$ per patient, resulting in a cost reduction of $9.9 \%$, a saving in absolute terms of $179,154.58 €$ (1006.49€ per patient).

Table 3 provides a summary of the costs involved for each of the drugs used in the sample. The total cost of the majority of the drugs shows a reduction of $20-98 \%$, which can be ascribed either to a reduction or discontinuance of the prescribed dosage. Among the notable results are: the cost of the prescription of Quetiapine, which during the period of 2013-2016 amounted to 242,287.37€ per year (4946.11€ per year per patient), and was reduced by $94.10 \%(14,289.75 €$ per year, $4104.12 €$ per year per patient). The total cost of prescriptions for Paliperidone $(113,284.36 €$ per year, $4714.87 €$ per year per patient) increased by $162 \%(297,452.8 €$ per year, $4282.34 €$ per year per patient); and Aripiprazole (62,296.64€ per year, $2807.18 €$ per year per patient) representing an increase of $35.6 \%(84,476.43 €$ per year, $2362.30 €$ per year per patient). The average cost involved in the pharmacotherapy of the patients treated with each of the drugs, whether as a monotherapy treatment or in combination with other antipsychotic drugs, showed a reduction of between $8 \%$ and $88 \%$.

Continuing our analysis of the influence on the pharmaceutical costs of an approach based on Precision Medicine, we will now analyse the costs associated with each of the demographic variables gathered in the present study. It is worth noting that the pharmaceutical costs of female patients $(n=69)$ were $172,496.2 €$ per year $(2574.6 €$ per year per patient), $44.3 \%$ less than the costs attributed to the male patients $(n=102)$, which reaches 309,775.3€/year (3480.6€ per year per patient, a decrease of $26.03 \%$ ). The pharmaceutical costs associated with the group of women were reduced by $4.4 \%(164,904.1 €$ per year, $2461.3 €$ per year per patient), while the reduction for men was $26.0 \%(229,256.9 €$ per year, $2575.9 €$ per year per patient). With regard to the diagnosis, the patients diagnosed with a subtype of schizotypal disorder (DSM-V, F25.?; n-107) amassed an average cost of $5987.9 €$ per year per patient $(53,890.9 €$ per year), followed by schizophrenic patients (DSM-V, F20.?; n=107) at $3202.9 €$ per year per patient $(342,708.9 €$ per year), while 
Table 3 Pharmaceutical Costs

\begin{tabular}{|c|c|c|c|c|c|c|}
\hline Antipsychotic & PrePGx (€) & PostPGx (€) & Variation (\%) & PrePGx ( $€ /$ Patient) & PostPGx (€/Patient) & Variation (\%) \\
\hline Olanzapine & $38,754.79$ & $18,438.89$ & -51.74 & 717.68 & 472.79 & -34.12 \\
\hline Aripiprazole & $62,296.64$ & $84,476.43$ & +20.16 & 1271.36 & 1919.92 & +51.01 \\
\hline Risperidone & $27,674.40$ & 354.12 & -98.70 & 588.82 & 177.06 & -69.99 \\
\hline Amisulpride & 4820.19 & 1222.15 & -74.65 & 370.78 & 174.59 & -52.91 \\
\hline Clozapine & $10,398.30$ & 5178.44 & -34.90 & 273.64 & 207.14 & -24.30 \\
\hline Paliperidone & || $3,284.5 \mid$ & $297,452.8$ & +162.57 & 2832.11 & 3913.85 & +38.20 \\
\hline Quetiapine & $242,287.37$ & $14,289.75$ & -94.10 & 3727.50 & 752.09 & -79.82 \\
\hline Asenapine & $14,289.75$ & 9526.50 & -33.33 & 752.09 & 635.10 & -15.56 \\
\hline Anual Total & $513,805.94$ & $462,618.92$ & -9.96 & 2886.55 & 2598.98 & -9.96 \\
\hline Triennium Total & I,798,320.80 & $1,619,166.20$ & -9.96 & $10,102.93$ & 9096.44 & -9.96 \\
\hline
\end{tabular}

Abbreviations: PrePGx, pharmaceutical cost associated with each drug before most of the population was pharmacogenotyped (2013-20I6); PostPGx, pharmaceutical cost associated with each drug after most of the population was pharmacogenotyped (2016-2019).

for bipolar patients (DSM-V, F31.?; N=23) the figures were $2490.4 €$ per year per patient $(57,279.4 €$ per year). Throughout the course of the study, the reduction in costs amounted to $68.6 \%$ (1879.3€ per year per patient, $16,913 €$ per year), 3.2\% (3101.1€ per year per patient, 331,813.0€ per year) and 52.2\% (1189.5€ per year per patient, 27,358.3€ per year), respectively. Separating the patients into four age brackets $(0-20, \mathrm{n}=0 ; 21-40, \mathrm{n}=44 ; 41-60, \mathrm{n}=91$; and $61+$, $\mathrm{n}=21$ ), we were able to confirm that patients aged between 21 and 40 accounted for pharmaceutical costs of $3431 €$ per year per patient $(151,005.3 €$ per year); the $41-60$ group had associated costs of $3047 €$ per year per patient $(277,330.3 €$ per year); and those over the age of sixty accounted for costs of $2573.2 €$ per year per patient $(54,037.3 €$ per year). These costs were reduced by $20.9 \%$ (2715.1€ per year per patient, $119,462.5 €$ per year), $18.1 \%$ (2494.9€ per year per patient, $227,035.8 €$ per year) and $4.3 \%$ (2463.5€ per year per patient, 51,734.4€ per year), respectively.

\section{Hospitalization Costs}

Prior to the application of the 5SPM Precision Medicine model (2013-2016), the 172 patients who had met the inclusion criteria accounted for 504 hospitalization stays (3 hospitalizations per patient) for a total of 4104 days hospitalized (24.87 hospitalization days per patient). The average duration of each hospital stay was 8.53 days. After adjusting the therapy according to the genotype of each patient, the total number of hospitalizations decreased by
$56.7 \%$ for a total of 218 hospitalizations (1.36 hospitalizations per patient, $-54.6 \%$ ) accounted for during the period of time between 2016 and 2019, for a total of 1561 days (14.45 hospitalization days per patient, $-41.9 \%$ ), with a reduction of $61.9 \%$ in the number of total days hospitalized. The average hospital stay was 7.09 days, 16.9\% less than the period of time between 2013 and 2016 (Figure 1).

Regarding reasons for hospital admission, the most common reason accounted for during the time frame 2013-2016 was discontinuance of treatment (147/504), followed by adverse effects (126/504) and clinical decompensation (112/504). Reasons for the other hospitalizations were either not specified $(35 / 504)$ or were not included among the three previously mentioned causes (84/504). Between 2016 and 2019, the number of hospitalizations due to discontinuance of treatment and the appearance of adverse effects decreased by $78 \%$ (32/218) and 88\% (15/ 218), respectively. Hospitalizations related to the decompensation of the underlying pathology decreased by $37 \%$ (70/218), and hospitalizations for unspecified reasons decreased by $17 \%$ (29/218). Hospitalizations not included among the three causes mentioned increased by $14 \%$ (72/ 232). The patients required an average of 1.6 hospitalizations due to a decompensation of the underlying pathology, which fell to $1.4(-8 \%), 1.7$ hospitalizations due to discontinuance of treatment, which dropped to $1.4(-19 \%)$, and 1.6 hospitalizations related to some type of adverse effect $1.3(-20 \%)$. Consequently, a change occurs in the profile of hospitalizations among the total population. 


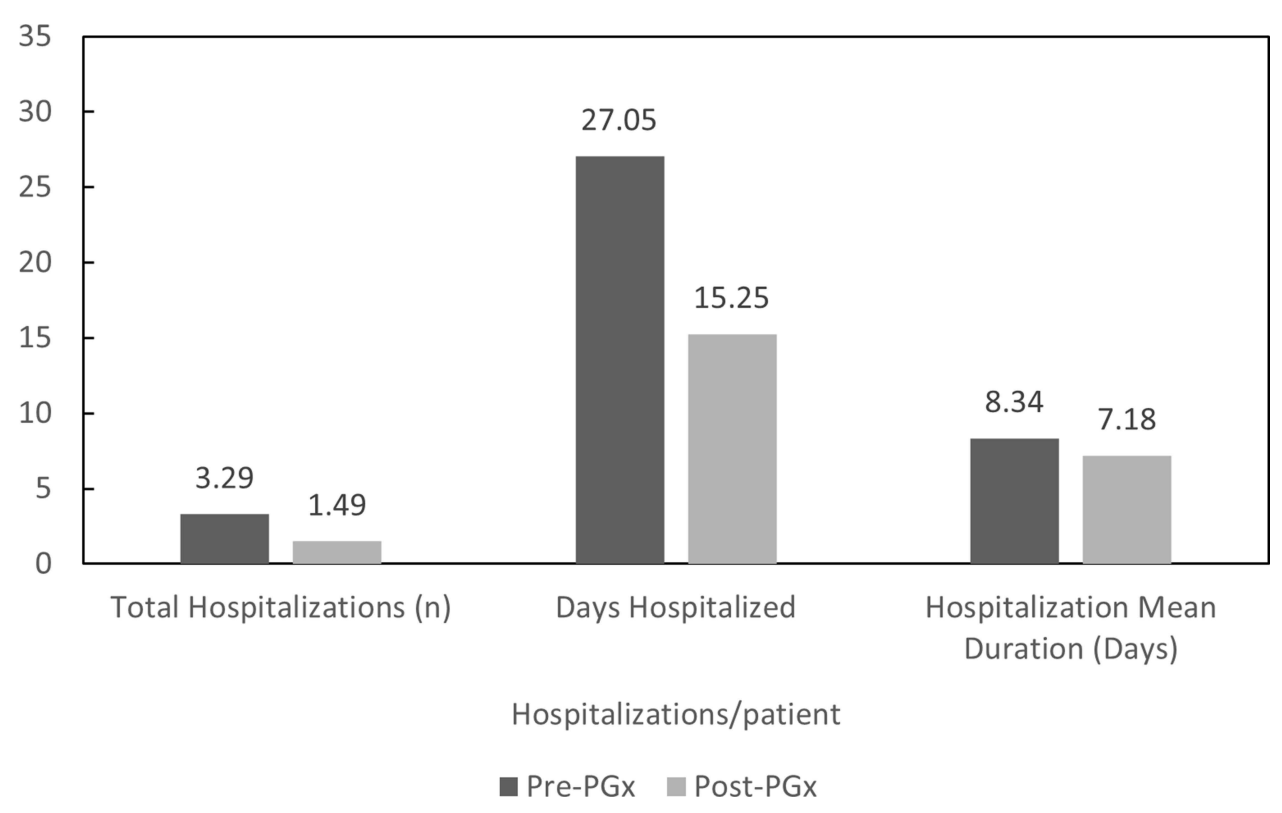

Figure I Total hospitalizations per patient, total days hospitalized per patient and hospitalization mean duration.

Abbreviations: PrePGx, pharmaceutical cost associated with each drug before most of the population was pharmacogenotyped (20I3-20I6); PostPGx, pharmaceutical cost associated with each drug after most of the population was pharmacogenotyped (2016-2019).

Whereas the primary motive was previously a discontinuance of treatment (29.2\%), followed by hospitalizations due to adverse effects $(25.0 \%)$ and decompensation of the underlying pathology $(22.2 \%)$, the latter has now become the primary motive $(32.1 \%)$, followed by discontinuance of treatment $(14.7 \%)$ and adverse effects (6.9\%) (Figure 2).
A study of the hospital stays (number, days, costs) based on different demographic variables (sex, diagnosis, age) demonstrates that, prior to the pharmacogenetic study, men were hospitalized more times (294 admissions = $215,502 €, 3.3=2421.4 €$ per patient $)$ than women $(209=$ $153,197 €, 3.1=2286.5 €$ per patient), with a longer duration (26.93 days versus 25.65 days). On average, the

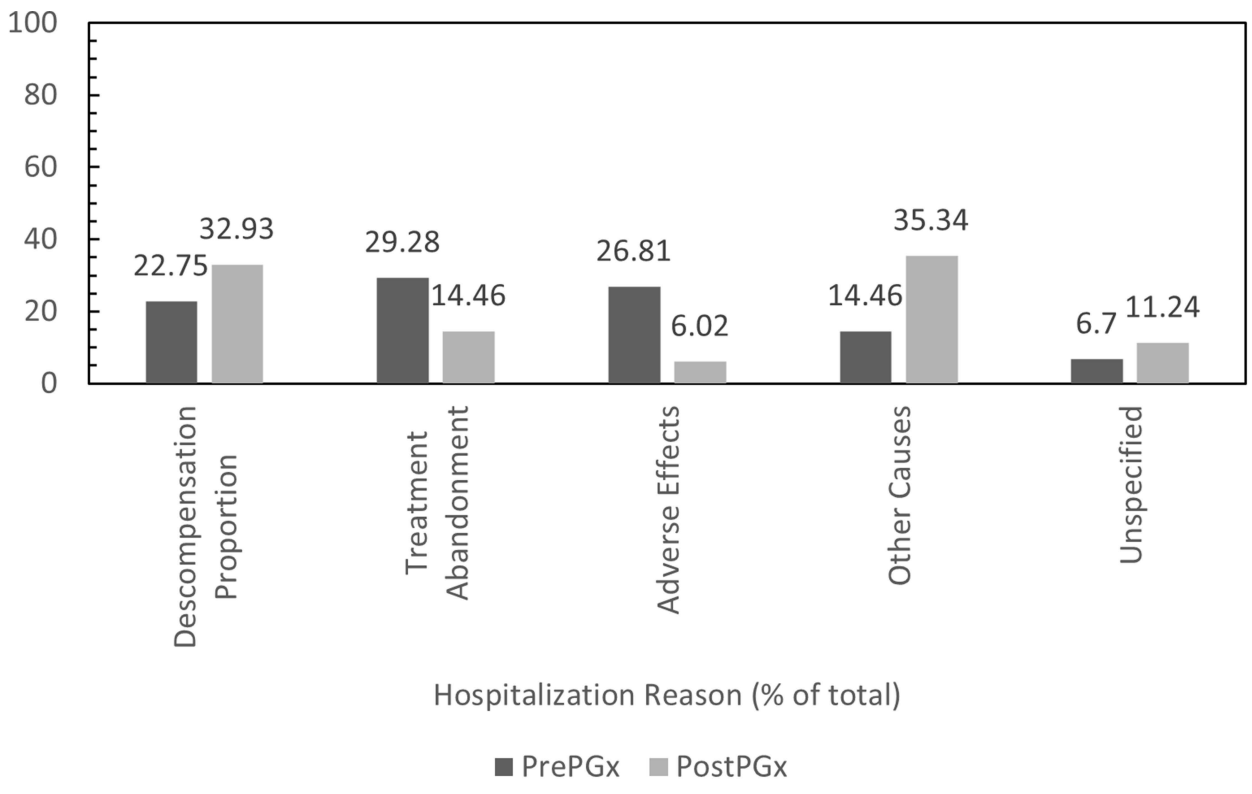

Figure 2 Hospitalization reasons.

Abbreviations: PrePGx, pharmaceutical cost associated with each drug before most of the population was pharmacogenotyped (20I3-20I6); PostPGx, pharmaceutical cost associated with each drug after most of the population was pharmacogenotyped (2016-2019). 
frequency of women hospitalized due to discontinuation of pharmacotherapy (1.8 versus 1.7) or to adverse effects (1.8 versus 1.6) was higher than for men, while admissions due to decompensation of the underlying pathology were much more frequent among men (1.7 versus 1.5) (Figure 3).

With regard to the diagnosis, patients diagnosed with a subtype of schizophrenia $(\mathrm{F} 20 ; \mathrm{n}=107)$ and with a subtype of personality disorder $(\mathrm{F} 60 ; \mathrm{n}=2)$ were hospitalized more frequently (3.6 and 4.5 admissions/patient, respectively), followed by patients with a schizophrenic disorder (F25, n=9; 3.4 admissions/patient) and a bipolar disorder ( $\mathrm{F} 31, \mathrm{n}=23 ; 2.6$ admissions/patient).

With regard to age, patients between 21 and 40 years of age $(n=44)$ were the most frequently hospitalized (3.7 admissions/patient) followed by the 41-60 age bracket (3.2 admissions/patient), while the group with the least number of admissions was 61 and over (2.9 admissions/ patient).

The reduction in the number of admissions, number of days in hospital, and the cost associated with the hospitalizations mirrors the figures calculated for the entire population, regardless of diagnosis, gender or age group $(50-60 \%)$.

\section{Financial Impact-Intervention}

Between 01 January 2013 and 30 June 2016, the pharmaceutical costs associated with the sample reached $1,798,320.80 €(10,102.93 €$ per patient) and the cost of all hospital stays (with an average cost of $733 €$ per admission and an average cost of $92.49 €$ per day of an inpatient hospital stay) is estimated between 369,432 and 37,9620 $€$ (2238.98-2300.73€ per patient). After applying the 5SPM methodology, between 01 July, 2016 and 31 December, 2019, total pharmaceutical costs decreased by $179,154.58 €$ (1006.49€ per patient), to a total of $1,619,166.20 €$ (9096.44€ per patient), and total hospital costs were calculated to have decreased between $144,392.5$ and $159,794 €$ (1336.97-1466€ per patient), for an estimated reduction in cost of 209,638-235,228€ ($772.98-963.76 €$ per patient), representing a total of 388 ,792.58-414,382.58€ (1779.47-1970.25€ per patient). Considering the fact that the cost of the pharmacogenetic

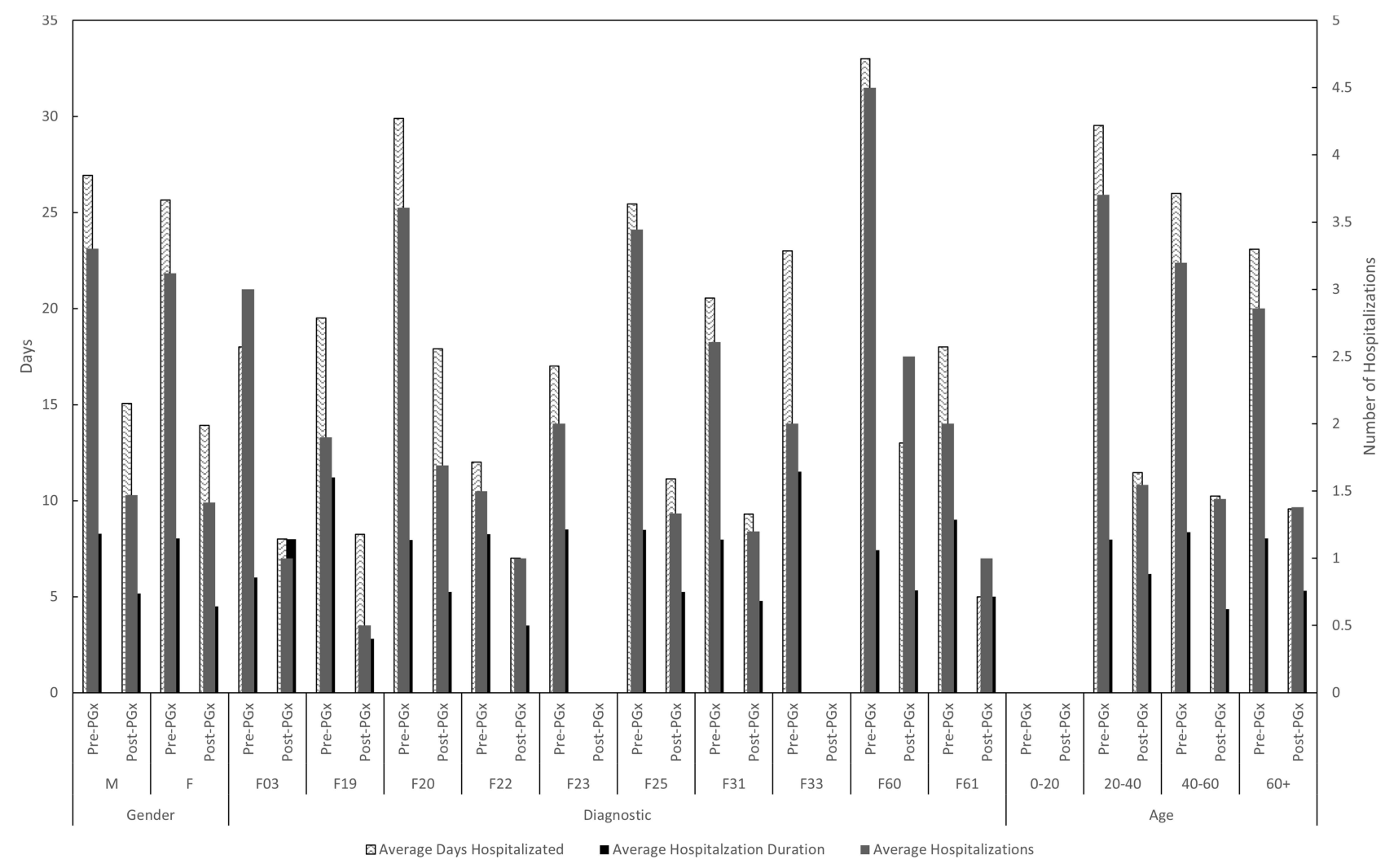

Figure 3 Average hospitalizations, average days hospitalized per patient and hospitalization duration for each demographic variable.

Abbreviations: PrePGx, pharmaceutical cost associated with each drug before most of the population was pharmacogenotyped (20I3-20I6); PostPGx, pharmaceutical cost associated with each drug after most of the population was pharmacogenotyped (2016-2019); M, male; F, female; F03, dementia; FI9, substance-related disorder; F20, schizophrenia; F22, persistent delusional disorder; F23, brief and acute psychotic disorder; F25, schizoaffective disorder; F3I, bipolar disorder; F33, major depressive disorder; F60, specific personality disorders; F6I, mixed personality disorder; F79, intellectual disability. 
study is $525 €$ per patient, the total investment for the present study of 172 patients was $90,300 €$, representing a net reduction between $298,492.58$ and $324,082.58 €$ (1254.47-1445.25€ per patient). An analysis of these figures should take the lifelong nature of the pharmacogenetic study into account. Therefore, for the 3-year period studied, the cost-benefit ratio is 3.31-3.59. Accounting for the particular situation of each patient, a reduction in total costs is noted in $67 \%$ of the patients (considering the pharmaceutical costs and those associated with inpatient hospital stay), which can be broken down to $51 \%$ of patients who saw a reduction in their pharmaceutical costs and $100 \%$ whose hospitalization costs decreased (Figure 4).

Regarding the influence of the concomitant pharmacotherapy, smoking and dietary habits on the metabolism of the antipsychotic pharmacotherapy - and therefore quite possibly on the therapeutic success and direct costs - no significant differences were found among the different groups studied, probably due to the very small sample size.

\section{Discussion}

The approach taken by the present study is to evaluate the cost-benefit ratio of the application of a pharmacogenetic analysis among long-term psychiatric patients who were found to have numerous adverse effects and only slight clinical improvement with the previously employed therapy. As a common factor, it should be noted that the majority of the patients included in the sample were polymedicated; in addition to their non-psychiatric concomitant therapy, different antipsychotics were simultaneously administered. To date, studies in this area have usually focused on the pharmacogenetic analysis directed at the metabolism of a single drug. Following this approach, the results have been disparate, although it is known that, under certain circumstances, this type of practice can be cost-effective and even cost-dominant. ${ }^{28}$ Our study demonstrated a positive cost-benefit ratio among twothirds of the patients, and that the main savings criteria were reached due to a decrease in the polytherapy and hospital stays. The consideration of polymedication in this type of study could be a positive aspect when searching for

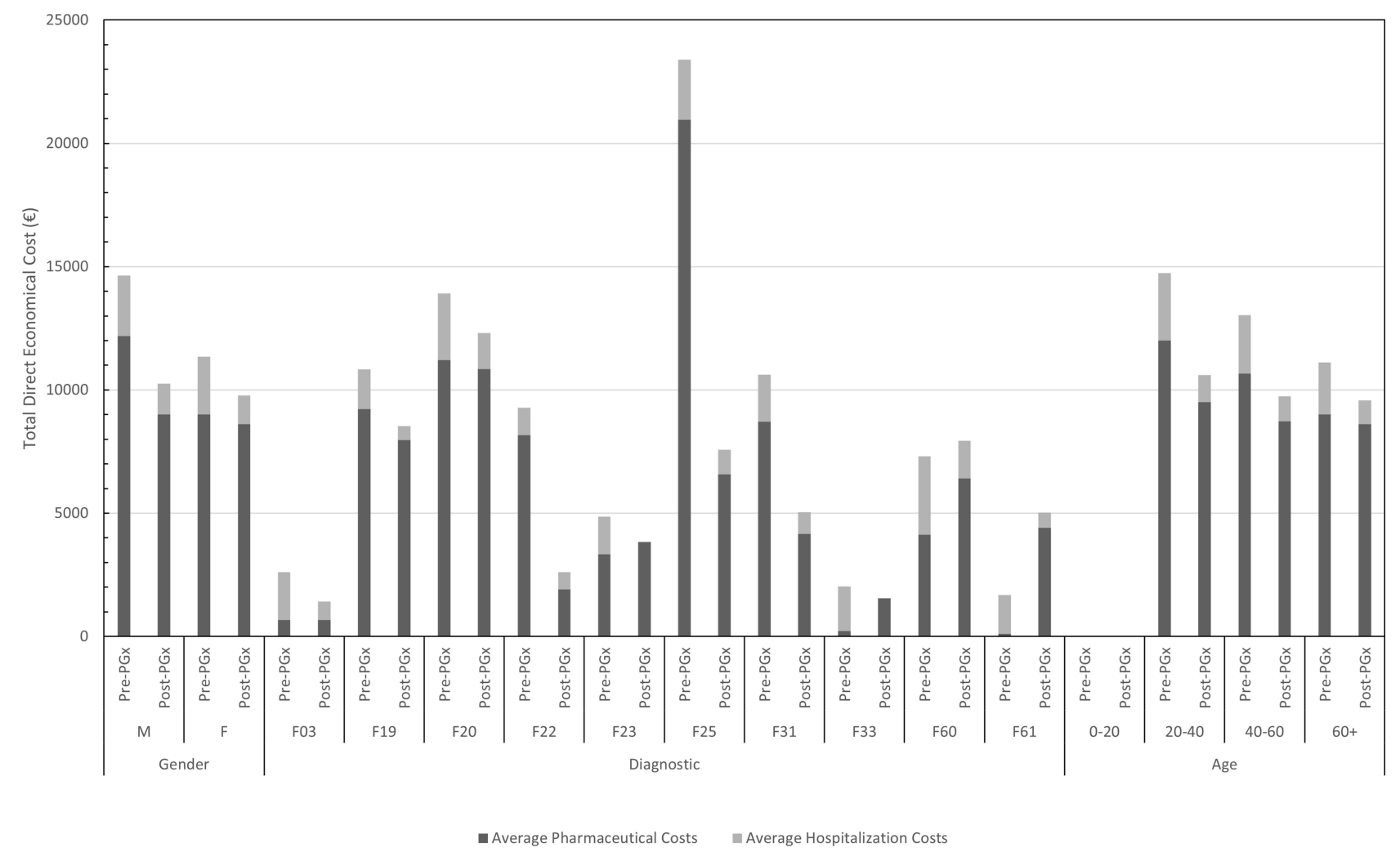

Figure 4 Average pharmaceutical costs and hospitalization costs per patient.

Abbreviations: PrePGx, pharmaceutical cost associated with each drug before most of the population was pharmacogenotyped (20I3-20I6); PostPGx, pharmaceutical cost associated with each drug after most of the population was pharmacogenotyped (2016-2019); M, male; F, female; F03, dementia; FI9, substance-related disorder; F20, schizophrenia; F22, persistent delusional disorder; F23, brief and acute psychotic disorder; F25, schizoaffective disorder; F3I, bipolar disorder; F33, major depressive disorder; F60, specific personality disorders; F6I, mixed personality disorder; F79, intellectual disability. 
robust evidence on the cost-effectiveness of this type of approach.

Our study corrected the pharmacotherapy in the 188 patients comprising our cohort, each of whom suffers from a serious, long-term mental illness. We applied the 5SPM Precision Medicine model, conditioned by the genotype of the members of the CYP450 system most relevantly involved in the metabolism of antipsychotics, and achieved a significant decrease in both pharmaceutical and hospitalization costs, which resulted in a decrease of total costs associated with oral medications metabolized by CYP1A2 (HI: 0.86) or a combination of these with other medications, as well as drugs that act on the most acute positive symptomatology but have a more complex profile of adverse effects. When the therapy was adjusted and greater effectiveness was achieved, their use was no longer required to assist in more complicated cases. Instead, the LAI psychotics Aripiprazole and Paliperidone, which have greater costs but facilitate adherence to treatment, were used and administered primarily in monotherapy in our sample. The pharmacogenetic profile of the population does not negatively interact with these drugs, resulting in fewer adverse effects and a consequent decrease in hospital expenses. Conversely, the elevated costs of the LAI antipsychotics, which increased in use during the period of time 20162019, could have partially masked the effect of the application of the 5SPM model on the pharmaceutical costs. This does not mean that LAI administration could be the general solution approach, given that patients were evaluated individually in order to avoid negative interaction between their therapy and genotype, which in turn involves greater pharmaceutical or hospital costs. Independently of the drugs used, during the period between 2016 and 2019 the average pharmaceutical costs were less than those incurred between 2013 and 2015 when compared to patient groups using the same pharmaceutical (whether in monotherapy or in combination with other drugs). As for the problems arising from polytherapy, the use of more than one drug was shown to be related to a higher number of hospital stays and economic costs. A correlation is also established between the number of pharmaceuticals employed and the average pharmaceutical cost, the number of hospital admissions, and the average number of days in hospital, suggesting that the decrease in this application, resulting from a personalized approach, can be linked to the decrease in pharmaceutical and hospital costs.

On the other hand, it is important to highlight that among the demographic groups with the lower pharmaceutical costs, the impact of the application of the precision medicine model was not as noticeable with regard to the pharmaceutical costs, possibly suggesting that in the specific case of patients in these groups, the original therapy was well established or, due to their clinical situation, there was either no need to use a large number of drugs or the therapeutic options were limited. This situation was not present when evaluating the hospital stays, whose variation (50-60\%) was not affected by any demographic variable, meaning that the hospital stays, and associated costs were reduced by a similar proportion among all patients.

Overall, the intervention was cost-dominant, and a substantial decrease was achieved in the number of hospital stays required by the patients for their clinical stability. It should be noted that the present study only evaluated direct costs and did not include indirect costs, which could be more complicated to compute. Moreover, given the manner in which clinical data are registered in different services, it was not possible to establish a comparison between the different states of the pathologies included in the study.

\section{Conclusion}

In conclusion, (1) the use of this methodology achieved a significant reduction in both pharmaceutical and hospitalization costs during the time frame 2016-2019 with respect to the time frame 2013-2015. Although future data should not be extrapolated, given that much depends on the individual evolution of the sample patients and on the appearance of new potentially stronger and cheaper therapeutic drugs and strategies, this finding is an especially positive point given the lifelong nature of the clinical intervention and the fact that the reduction in costs obtained greatly exceeds the investment required to perform a pharmacogenetic analysis. (2) Although depot injections are especially costly, they are sufficiently effective for the wide spectrum of symptomatology associated with psychotic disorders so as to be administered in monotherapy or accompanied by other drugs in lower doses, which, if the drug profile of the sample is known, makes it easier to avoid the appearance of adverse effects and consequent hospital stays, and to improve adherence to treatment. (3) The 
rational focus has resulted in a reduction in the number of drugs required to control the psychotic pathology, reducing the risk of adverse effects and improving adherence to treatment, leading to a decrease in hospital costs for admission due to adverse effects that cannot be remedied with outpatient treatment or to discontinuance of treatment, as well as a decrease in pharmaceutical costs resulting from a lower number of polytherapy cases or from a variance in the dosage used by the patient.

These conclusions should be considered with an awareness of the limitations of this study, in particular the small sample size. The data used to carry out the study were taken from the application of these pharmacogenetic tools in routine clinical practice and requested in accordance with the criteria of the medical prescriber conforming to the clinical evaluation of the patient (following strict guidelines conveyed to psychiatric specialists during special courses organized by the public administration). Each decision was made on an individual basis and agreed to by a multidisciplinary team (psychiatrists, psychologists, nurses, social workers and clinical biochemists), and only those patients who were deemed to obtain a clinical advantage were selected for this methodology. On the other hand, the decision to introduce this methodology coincided with an increase in the use of LAI antipsychotics, which facilitated stronger therapeutic adherence, and therefore less clinical failure, being this situation a possible confounder.

\section{Disclosure}

The authors report no conflicts of interest in this work.

\section{References}

1. WHO | Mental health action plan 2013-2020. WHO [Internet]. World Health Organization; 2015 [cited October 16, 2020]. Available from: http://www.who.int/entity/mental_health/publications/action_plan/en/ index.html. Accessed July 28, 2021.

2. McGrath J, Saha S, Chant D, Welham J. Schizophrenia: a concise overview of incidence, prevalence, and mortality. Epidemiol Rev. 2008;30(1):67-76. doi:10.1093/epirev/mxn001

3. NIMH. Schizophrenia [Internet]. [cited March 7, 2021]. Available from: https://www.nimh.nih.gov/health/statistics/schizophrenia.shtml. Accessed July 28, 2021.

4. Lally J, MacCabe JH. Antipsychotic medication in schizophrenia: a review. Br Med Bull. 2015;114(1):169-179. doi:10.1093/bmb/ldv017

5. Ozomaro U, Wahlestedt $C$, Nemeroff $C B$. Personalized medicine in psychiatry: problems and promises. BMC Med. 2013;11(1):1-35. doi:10.1186/1741-7015-11-132

6. Jin H, Mosweu I. The societal cost of schizophrenia: a systematic review. Pharmacoeconomics. 2017;35:25-42. doi10.1007/s40273-0160444-6
7. Unim B, Pitini E, De Vito C, D'Andrea E, Marzuillo C, Villari P. Cost-effectiveness of RAS genetic testing strategies in patients with metastatic colorectal cancer: a systematic review. Value Health. 2020;23:114-126. doi:10.1016/j.jval.2019.07.009

8. Martín AS, Gómez AI, García-Berrocal B, et al. Dose reduction of efavirenz: an observational study describing cost-effectiveness, pharmacokinetics and pharmacogenetics. Pharmacogenomics. 2014;15:997-1006. doi:10.2217/pgs.14.48

9. Borse MS, Dong OM, Polasek MJ, Farley JF, Stouffer GA, Lee CR. CYP2C19-guided antiplatelet therapy: a cost-effectiveness analysis of 30-day and 1-year outcomes following percutaneous coronary intervention. Pharmacogenomics. 2017;18(12):1155-1166. doi:10.2217/pgs-2017-0075

10. Zhu Y, Swanson KM, Rojas RL, et al. Systematic review of the evidence on the cost-effectiveness of pharmacogenomics-guided treatment for cardiovascular diseases. Genet Med. 2020;22:475-486.

11. Pink J, Pirmohamed M, Lane S, Hughes DA. Cost-effectiveness of pharmacogenetics-guided warfarin therapy vs. Alternative anticoagulation in atrial fibrillation. Clin Pharmacol Ther. 2014;95 (2):199-207. doi:10.1038/clpt.2013.190

12. Berm EJJ, Hak E, Postma M, et al. Effects and cost-effectiveness of pharmacogenetic screening for CYP2D6 among older adults starting therapy with nortriptyline or venlafaxine: study protocol for a pragmatic randomized controlled trial (CYSCEtrial). Trials. 2015;16(1):1-8. doi:10.1186/s13063-015-0561-0

13. Verhoef TI, Redekop WK, Van Schie RM, et al. Cost-effectiveness of pharmacogenetics in anticoagulation: international differences in healthcare systems and costs. Pharmacogenomics. 2012;13:1405-1417. doi:10.2217/pgs.12.124

14. Sluiter RL, Kievit W, Van Der Wilt GJ, et al. Cost-effectiveness analysis of genotype-guided treatment allocation in patients with alcohol use disorders using naltrexone or acamprosate, using a modeling approach. Eur Addict Res. 2018;24(5):245-254. doi: $10.1159 / 000494127$

15. De Monaco A, Berretta M, Pugliese S, Valente D, Ciaffarafa S, Di Francia R. Evaluation of genotyping methods and the relative cost of pharmacogenomics. Eur Rev Med Pharmacol Sci. 2014;18 (14):2084-2087.

16. Plöthner M, Ribbentrop D, Hartman J-P, Frank M. Cost-effectiveness of pharmacogenomic and pharmacogenetic test-guided personalized therapies: a systematic review of the approved active substances for personalized medicine in Germany. Adv Ther. 2016;33 (9):1461-1480. doi:10.1007/s12325-016-0376-8

17. Plumpton CO, Roberts D, Pirmohamed M, Hughes DA. A systematic review of economic evaluations of pharmacogenetic testing for prevention of adverse drug reactions. PharmacoEconomics. 2016;34 (8):771-793. doi:10.1007/s40273-016-0397-9

18. Fragoulakis V, Bartsakoulia M, Díaz-Villamarín X, et al. Costeffectiveness analysis of pharmacogenomics-guided clopidogrel treatment in Spanish patients undergoing percutaneous coronary intervention. Pharmacogenomics J. 2019;19(5):438-445. doi:10.1038/s41397-019-0069-1

19. Martin A, Downing J, Maden M, et al. An assessment of the impact of pharmacogenomics on health disparities: a systematic literature review. Pharmacogenomics. 2017;18(16):1541-1550. doi:10.2217/ pgs-2017-0076

20. Groessl EJ, Tally SR, Hillery N, Maciel A, Garces JA. Costeffectiveness of a pharmacogenetic test to guide treatment for major depressive disorder. J Manag Care Spec Pharm. 2018;24(8):726-734.

21. Corponi F, Fabbri C, Serretti A. Pharmacogenetics and depression: a critical perspective. Psychiatry Investig. 2019;16(9):645-653. doi:10.30773/pi.2019.06.16

22. van Westrhenen R, Aitchison KJ, Ingelman-Sundberg M, Jukić MM. Pharmacogenomics of antidepressant and antipsychotic treatment: how far have we got and where are we going? Front Psychiatry. 2020;11:94. doi:10.3389/fpsyt.2020.00094 
23. Li KX, Loshak H. Pharmacogenomic testing in depression: a review of clinical effectiveness, cost-effectiveness, and guidelines [Internet] Pharmacogenomic testing in depression: a review of clinical effectiveness, cost-effectiveness, and guidelines. Can Agency Drugs Technol Health. 2020 [cited December 7, 2020]. Available from. http://www.ncbi.nlm.nih.gov/pubmed/33074624. Accessed July 28, 2021.

24. Rosenblat JD, Lee Y, McIntyre RS. Does pharmacogenomic testing improve clinical outcomes for major depressive disorder? a systematic review of clinical trials and cost-effectiveness studies. J Clin Psychiatry. 2017;78:720-729. doi10.4088/JCP.15r10583

25. Peterson K, Dieperink E, Ferguson L, Anderson J, Helfand M. Evidence brief: the comparative effectiveness, harms, and cost-effectiveness of pharmacogenomics-guided antidepressant treatment versus usual care for major depressive disorder [Internet]. VA Evidence-based Synthesis Program Evidence Briefs; 2011 [cited December 7, 2020]. Available from: http://www.ncbi.nlm.nih.gov/ pubmed/27606389. Accessed July 28, 2021.

26. Fabbri C, Kasper S, Zohar J, et al. Cost-effectiveness of genetic and clinical predictors for choosing combined psychotherapy and pharmacotherapy in major depression. $J$ Affect Disord. 2020;279:722-729. Elsevier BV.

27. Girardin FR, Poncet A, Perrier A, et al. Cost-effectiveness of HLA-DQB1/HLA-B pharmacogenetic-guided treatment and blood monitoring in US patients taking clozapine. Pharmacogenomics J. 2019;19(2):211-218. doi:10.1038/s41397-017-0004-2
28. Verbelen M, Weale ME, Lewis CM. Cost-effectiveness of pharmacogenetic-guided treatment: are we there yet? Pharmacogenomics J. 2017;17:395-402. doi10.1038/tpj.2017.21

29. Kim E, Levy R, Pikalov A. Personalized treatment with atypical antipsychotic medications. Adv Ther. 2007;24:721-740. doi:10.1007/BF02849966

30. Moore T, Hill A, Panguluri S. Pharmacogenomics in psychiatry: implications for practice. Recent Pat Biotechnol. 2014;8 (2):152-159. doi:10.2174/1872208309666140904113615

31. Carrascal-Laso L, Franco-Martín MÁ, García-Berrocal MB, et al. Application of a pharmacogenetics-based precision medicine model (5SPM) to psychotic patients that presented poor response to neuroleptic therapy. J Pers Med. 2020;10(4):1-16. doi:10.3390/ jpm10040289

32. de Leon J, Susce MT, Murray-Carmichael E. The AmpliChipTM CYP450 genotyping test. Mol Diagn Ther. 2012;10(3):135-151. doi:10.1007/BF03256453

33. Gaedigk A, Whirl-Carrillo M, Pratt VM, Miller NA, Klein TE. PharmVar and the landscape of pharmacogenetic resources. Clin Pharmacol Ther. 2020;107(1):43-46. doi:10.1002/cpt.1654
Pharmacogenomics and Personalized Medicine

\section{Publish your work in this journal}

Pharmacogenomics and Personalized Medicine is an international, peer-reviewed, open access journal characterizing the influence of genotype on pharmacology leading to the development of personalized treatment programs and individualized drug selection for improved safety, efficacy and sustainability. This journal is indexed
Dovepress

on the American Chemical Society's Chemical Abstracts Service (CAS). The manuscript management system is completely online and includes a very quick and fair peer-review system, which is all easy to use. Visit http://www.dovepress.com/testimonials.php to read real quotes from published authors. 